-Supporting Information: Part A-

\title{
A Rapid Access to the Structural Core of Aflavinines via Stereoselective Tandem Intramolecular Diels-Alder Cycloaddition Controlled by the Allylic 1,3-Strain
}

\author{
Minmi Jo, ${ }^{\dagger}$ Dongjoo Lee, ${ }^{* \star}$ and Young-Shin Kwak ${ }^{* \dagger}$ \\ ${ }^{\dagger}$ College of Pharmacy, Korea University, Sejong 30019, Republic of Korea \\ College of Pharmacy and Research Institute of Pharmaceutical Science and Technology \\ (RIPST), Ajou University, Suwon 16499, Republic of Korea \\ E-mail: youngshin@korea.ac.kr \\ E-mail: dongjoo@ajou.ac.kr \\ Part A (S01-S14) \\ Experimental Procedures and Product Characterization
}

I. Materials and Methods

S02

II. Full Experimental Procedures and Analytical Data of Compounds

S03-S13

III. References

S14 


\section{Materials and Methods}

General Methods: Except as otherwise indicated, reactions were carried out under argon atmosphere in flame- or oven-dried glassware. In aqueous work-up, all organic solutions were dried over sodium sulfate $\left(\mathrm{Na}_{2} \mathrm{SO}_{4}\right)$ or magnesium sulfate $\left(\mathrm{MgSO}_{4}\right)$, and filtered prior to rotary evaporation at water aspirator pressure. Reactions were monitored by thin layer chromatography (TLC) with $0.25-\mathrm{mm}$ E. Merck pre-coated silica gel plates (Kieselgel 60F 254 , Merck). Spots were detected by viewing under a UV light, colorizing with charring after dipping in $p$-anisaldehyde solution with acetic acid and sulfuric acid and ethanol, or in $\mathrm{KMnO}_{4}$ solution with sulfuric acid and ethanol, or ceric ammonium molybdate solution with sulfuric acid and ethanol. Silica gel for flash chromatography (particle size $0.040-0.063 \mathrm{~mm}$ ) was supplied by E. Merck. Yields refer to chromatographically and spectroscopically pure compounds unless otherwise noted.

Materials: All commercial reagents and solvents were purchased from Sigma Aldrich Co. or Tokyo Chemical Industry (TCI) and used as received with the following exceptions. All solvents were freshly purified and dried by standard techniques just before use. Tetrahydrofuran (THF) was distilled from sodium/benzophenone. Dichloromethane $\left(\mathrm{CH}_{2} \mathrm{Cl}_{2}\right)$, acetonitrile $(\mathrm{MeCN}), N, N$-dimethylformamide $\left(\mathrm{Me}_{2} \mathrm{NC}(=\mathrm{O}) \mathrm{H}\right)$, benzene $\left(\mathrm{C}_{6} \mathrm{H}_{6}\right)$ and toluene $\left(\mathrm{C}_{7} \mathrm{H}_{8}\right)$ were distilled from calcium hydride $\left(\mathrm{CaH}_{2}\right)$. Methanol $(\mathrm{MeOH})$ was distilled from magnesium sulfate $\left(\mathrm{MgSO}_{4}\right)$.

Instrumentation: ${ }^{1} \mathrm{H}$ and ${ }^{13} \mathrm{C}$ spectra were recorded on Bruker DPX $600 \mathrm{MHz}$ spectrometer. Chemical shifts are reported as $\delta$ value relative to internal chloroform $\left(\delta 7.26\right.$ for ${ }^{1} \mathrm{H}$ and $\delta 77.0$ for ${ }^{13} \mathrm{C}$ ). Data are represented as follows: chemical shift, multiplicity $(\mathrm{s}=$ singlet, $\mathrm{d}=$ doublet, $\mathrm{t}=$ triplet, $\mathrm{q}=$ quartet, $\mathrm{m}=$ multiplet, $\mathrm{br}=$ broad), coupling constant in $\mathrm{Hz}$, and integration. High resolution mass spectra (HRMS) were recorded on Agilent 6530 Q-TOF or Thermo Scientific Q Exactive HF-X Hybrid Quadrupole-Orbitrap mass spectrometer. High resolution values are calculated to four decimal places from the molecular formula, all found values being within a tolerance of $5 \mathrm{ppm}$. Melting point (m.p.) was obtained using Thermo IA9100 melting point apparatus. 


\section{Full Experimental Procedures and Analytical Data of Compounds}

\section{1-(3-Methylfuran-2-yl)-6-(trimethylsilyl)hex-5-yn-1-one [20]}

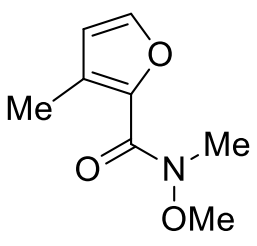

16
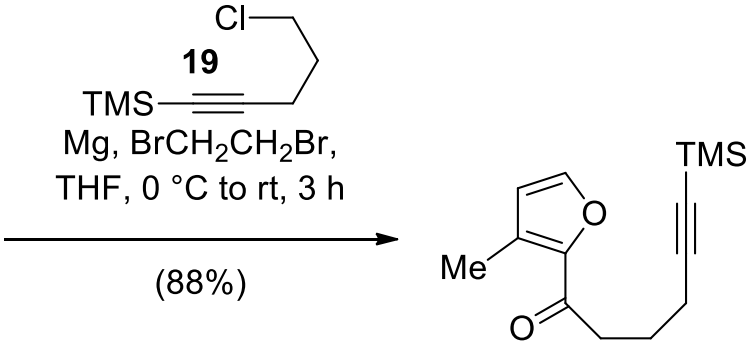

20

To a cooled $\left(0^{\circ} \mathrm{C}\right)$ stirred solution of $16^{1}(3.30 \mathrm{~g}, 19.5 \mathrm{mmol})$ in anhydrous THF $(15 \mathrm{~mL})$ was added dropwise Grignard reagent solution, which was prepared in situ by the slow addition of 1,2-dibromoethane $(420 \mu \mathrm{L}, 4.88 \mathrm{mmol})$ into the suspension of magnesium turnings $(1.42 \mathrm{~g}$, $58.5 \mathrm{mmol}$ ) and (5-chloropent-1-yn-1-yl)trimethylsilane (19) (4.09 g, $23.4 \mathrm{mmol})$ in anhydrous THF $(25 \mathrm{~mL}, 0.78 \mathrm{M})$ for 2 hours at room temperature under argon atmosphere. The reaction mixture was stirred at room temperature for 3 hours and cooled to $0{ }^{\circ} \mathrm{C}$ under argon atmosphere, then quenched with $\mathrm{H}_{2} \mathrm{O}(20 \mathrm{~mL})$ and diluted with EtOAc $(30 \mathrm{~mL})$. The $\mathrm{pH}$ of the solution was adjusted to 2 by the addition of $1 \mathrm{~N} \mathrm{HCl}$ solution dropwise. The layers were separated and the aqueous layer was extracted with EtOAc $(2 \times 30 \mathrm{~mL})$. The combined organic layers were washed successively with $\mathrm{H}_{2} \mathrm{O}(5 \mathrm{~mL})$ and brine $(5 \mathrm{~mL})$, dried over anhydrous $\mathrm{MgSO}_{4}$, filtered and concentrated in vacuo. The residue was purified by column chromatography (silica gel, heptane:EtOAc $=40: 1)$ to afford $20(4.26 \mathrm{~g}, 88 \%)$ as a colorless oil.

${ }^{1} \mathrm{H} \mathrm{NMR}\left(600 \mathrm{MHz}, \mathrm{CDCl}_{3}\right) \delta 7.39(\mathrm{~s}, 1 \mathrm{H}), 6.38(\mathrm{~s}, 1 \mathrm{H}), 2.96(\mathrm{t}, J=7.2 \mathrm{~Hz}, 2 \mathrm{H}), 2.39(\mathrm{~s}, 3 \mathrm{H})$, $2.34(\mathrm{t}, J=7.0 \mathrm{~Hz}, 2 \mathrm{H}), 1.92(\mathrm{~m}, 2 \mathrm{H}), 0.14(\mathrm{~s}, 9 \mathrm{H}) ;{ }^{13} \mathrm{C} \mathrm{NMR}\left(150 \mathrm{MHz}, \mathrm{CDCl}_{3}\right) \delta 191.0$, 148.7, 144.2, 130.3, 116.0, 106.8, 85.4, 37.9, 22.8, 19.6, 12.0, 0.3; HRMS (ESI-quadrupole) $m / z:[\mathrm{M}+\mathrm{H}]^{+}$Calcd for $\mathrm{C}_{14} \mathrm{H}_{21} \mathrm{O}_{2} \mathrm{Si}$ 249.1310; Found 249.1306.

( \pm )-1-(3-Methylfuran-2-yl)-6-(trimethylsilyl)hex-5-yn-1-ol $[( \pm)-21]$<smiles>CCCCCC#CCCCC(=O)c1occc1C</smiles>

20

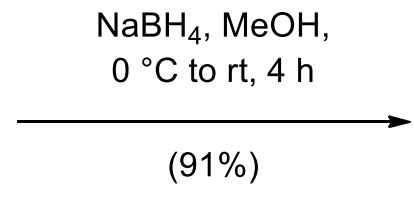

20 (1.88 g, $7.57 \mathrm{mmol})$ in anhydrous $\mathrm{MeOH}(25 \mathrm{~mL}, 0.30$ M) was added $\mathrm{NaBH}_{4}(400 \mathrm{mg}, 10.6 \mathrm{mmol})$ portionwise under argon atmosphere. The reaction mixture was gradually warmed to room temperature over the period of 4 hours, then quenched with saturated aqueous $\mathrm{NH}_{4} \mathrm{Cl}$ solution $(10 \mathrm{~mL})$ and diluted with EtOAc $(20 \mathrm{~mL})$. The layers were separated and the aqueous layer was extracted with EtOAc $(2 \times 20 \mathrm{~mL})$. The combined 
organic layers were washed successively with $\mathrm{H}_{2} \mathrm{O}(5 \mathrm{~mL})$ and brine $(5 \mathrm{~mL})$, dried over anhydrous $\mathrm{MgSO}_{4}$ and concentrated in vacuo. The residue was purified by column chromatography (silica gel, heptane:EtOAc $=20: 1)$ to afford $( \pm)-\mathbf{2 1}(1.73 \mathrm{~g}, 91 \%)$ as a colorless oil.

${ }^{1} \mathrm{H}$ NMR $\left(600 \mathrm{MHz}, \mathrm{CDCl}_{3}\right) \delta 7.27(\mathrm{~d}, J=1.2 \mathrm{~Hz}, 1 \mathrm{H}), 6.18(\mathrm{~d}, J=1.2 \mathrm{~Hz}, 1 \mathrm{H}), 4.72(\mathrm{q}, J=$ $6.6 \mathrm{~Hz}, 1 \mathrm{H}), 2.23(\mathrm{dt}, J=7.1,2.6 \mathrm{~Hz}, 2 \mathrm{H}), 2.04(\mathrm{~s}, 3 \mathrm{H}), 2.03-1.89(\mathrm{~m}, 2 \mathrm{H}), 1.78(\mathrm{~d}, J=5.4$ $\mathrm{Hz}, 1 \mathrm{H}), 1.64-1.55(\mathrm{~m}, 1 \mathrm{H}), 1.50-1.42(\mathrm{~m}, 1 \mathrm{H}), 0.14(\mathrm{~s}, 9 \mathrm{H}) ;{ }^{13} \mathrm{C} \mathrm{NMR}\left(125 \mathrm{MHz}, \mathrm{CDCl}_{3}\right) \delta$ 150.6, 141.0, 116.3 113.0, 107.0, 84.9, 65.7, 34.7, 24.7, 19.6, 9.7, 0.14; HRMS (ESI-TOF) $\mathrm{m} / \mathrm{z}$ : $[\mathrm{M}+\mathrm{H}]^{+}$Calcd for $\mathrm{C}_{14} \mathrm{H}_{23} \mathrm{O}_{2} \mathrm{Si} 251.1467$; Found 251.2060.

( \pm )-(R)-1-(3-Methylfuran-2-yl)hex-5-yn-1-ol [( \pm )-22]

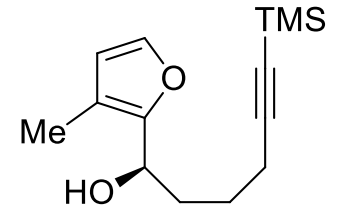

$( \pm)-21$

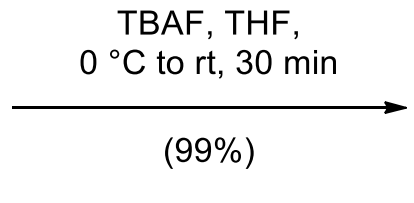

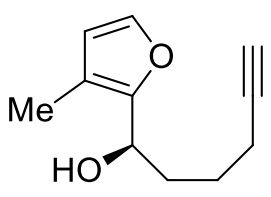

$( \pm)-22$

To a cooled $\left(0{ }^{\circ} \mathrm{C}\right)$ stirred solution of $( \pm)-21(780 \mathrm{mg}, 3.11 \mathrm{mmol})$ in THF $(15 \mathrm{~mL}, 0.21 \mathrm{M})$ was dropwise added tetrabutylammonium fluoride (TBAF, $3.72 \mathrm{~mL}, 1 \mathrm{M}$ solution in THF, 3.72 mmol) under argon atmosphere. The reaction mixture was gradually warmed to room temperature over the period of 30 minutes, then quenched with saturated aqueous $\mathrm{NH}_{4} \mathrm{Cl}$ solution $(5 \mathrm{~mL})$ and diluted with EtOAc $(15 \mathrm{~mL})$. The layers were separated and the aqueous layer was extracted with EtOAc $(2 \times 15 \mathrm{~mL})$. The combined organic layers were washed successively with $\mathrm{H}_{2} \mathrm{O}(5 \mathrm{~mL})$ and brine $(5 \mathrm{~mL})$, dried over anhydrous $\mathrm{MgSO}_{4}$ and concentrated in vacuo. The residue was purified by column chromatography (silica gel, heptane:EtOAc = 10:1) to afford $( \pm)-22(1.10 \mathrm{~g}, 99 \%)$ as a colorless oil.

${ }^{1} \mathrm{H}$ NMR $\left(600 \mathrm{MHz}, \mathrm{CDCl}_{3}\right) \delta 7.28(\mathrm{~d}, J=1.2 \mathrm{~Hz}, 1 \mathrm{H}), 6.19(\mathrm{~d}, J=1.2 \mathrm{~Hz}, 1 \mathrm{H}), 4.72(\mathrm{q}, J=$ $7.2 \mathrm{~Hz}, 1 \mathrm{H}), 2.22(\mathrm{td}, J=7.8,1.2 \mathrm{~Hz}, 2 \mathrm{H}), 2.04(\mathrm{~s}, 3 \mathrm{H}), 2.09-1.99(\mathrm{~m}, 1 \mathrm{H}), 1.99-1.91(\mathrm{~m}, 1 \mathrm{H})$, $1.95(\mathrm{t}, J=2.6 \mathrm{~Hz}, 1 \mathrm{H}), 1.76(\mathrm{~d}, J=5.4 \mathrm{~Hz}, 1 \mathrm{H}), 1.67-1.57(\mathrm{~m}, 1 \mathrm{H}), 1.53-1.41(\mathrm{~m}, 1 \mathrm{H}) ;{ }^{13} \mathrm{C}$ NMR $\left(150 \mathrm{MHz}, \mathrm{CDCl}_{3}\right) \delta 150.5,141.1,116.4,113.0,84.1,68.6,65.6,34.7,24.6,18.2,9.7$; HRMS (ESI-TOF) $m / z$ : [M+H] $]^{+}$Calcd for $\mathrm{C}_{11} \mathrm{H}_{15} \mathrm{O}_{2}$ 179.1072; Found 179.1018.

\section{$( \pm)-(R)$-Triisopropyl((1-(3-methylfuran-2-yl)hex-5-yn-1-yl)oxy)silane [( \pm )-23]}

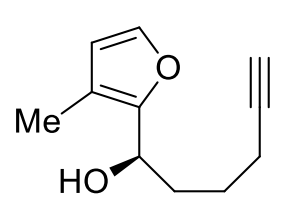

$( \pm)-22$

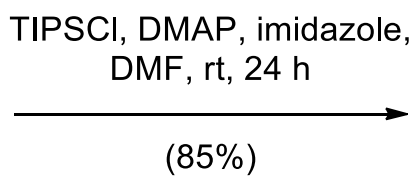

TIPSO

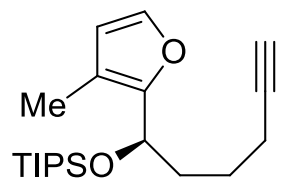

$( \pm)-23$

To a stirred solution of $( \pm)-22(550 \mathrm{mg}, 3.09 \mathrm{mmol})$ in DMF $(10 \mathrm{~mL}, 0.31 \mathrm{M})$ was successively added imidazole (490 mg, $7.14 \mathrm{mmol}$ ), 4-dimethylaminopyridine (DMAP, $110 \mathrm{mg}, 0.930 \mathrm{mmol}$ ) 
and triisopropylsilyl chloride (TIPSCl, $1.38 \mathrm{~mL}, 6.52 \mathrm{mmol}$ ) at room temperature under argon atmosphere. The reaction mixture was stirred at the same temperature for 24 hours, then quenched with saturated aqueous $\mathrm{NH}_{4} \mathrm{Cl}$ solution $(5 \mathrm{~mL})$ and diluted with EtOAc $(15 \mathrm{~mL})$. The layers were separated and the aqueous layer was extracted with EtOAc $(2 \times 15 \mathrm{~mL})$. The combined organic layers were washed successively with $\mathrm{H}_{2} \mathrm{O}(5 \mathrm{~mL})$ and brine $(5 \mathrm{~mL})$, dried over anhydrous $\mathrm{MgSO}_{4}$ and concentrated in vacuo. The residue was purified by column chromatography (silica gel, heptane:EtOAc $=50: 1)$ to afford $( \pm)-23(880 \mathrm{mg}, 85 \%)$ as a colorless oil.

${ }^{1} \mathrm{H}$ NMR $\left(600 \mathrm{MHz}, \mathrm{CDCl}_{3}\right) \delta 7.24(\mathrm{~d}, J=1.2 \mathrm{~Hz}, 1 \mathrm{H}), 6.13(\mathrm{~d}, J=1.2 \mathrm{~Hz}, 1 \mathrm{H}), 4.80(\mathrm{t}, J=$ $7.2 \mathrm{~Hz}, 1 \mathrm{H}), 2.18-2.13(\mathrm{~m}, 2 \mathrm{H}), 2.02(\mathrm{~s}, 3 \mathrm{H}), 2.00-1.87(\mathrm{~m}, 2 \mathrm{H}), 1.93(\mathrm{t}, J=2.6 \mathrm{~Hz}, 1 \mathrm{H}), 1.54$ $1.45(\mathrm{~m}, 1 \mathrm{H}), 1.44-1.33(\mathrm{~m}, 1 \mathrm{H}), 1.06-0.93(\mathrm{~m}, 21 \mathrm{H}) ;{ }^{13} \mathrm{C} \mathrm{NMR}\left(150 \mathrm{MHz}, \mathrm{CDCl}_{3}\right) \delta 151.3$, $140.4,115.2,112.8,84.3,68.4,66.7,36.7,24.4,18.3,18.0,17.8,12.3,10.0$; HRMS (ESIquadrupole) $m / z$ : $[\mathrm{M}+\mathrm{H}]^{+}$Calcd for $\mathrm{C}_{20} \mathrm{H}_{35} \mathrm{O}_{2} \mathrm{Si} 335.2406$; Found 335.2401.

\section{( \pm )-Methyl 7-(3-Methylfuran-2-yl)-7-((triisopropylsilyl)oxy)hept-2-ynoate $[( \pm)-24]$}

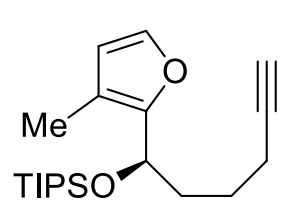

$( \pm)-23$

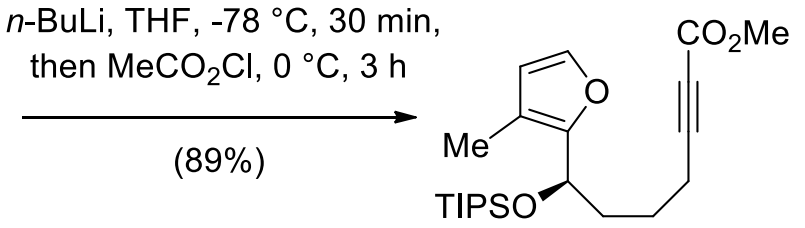

$( \pm)-24$

To a cooled $\left(-78^{\circ} \mathrm{C}\right)$ stirred solution of $( \pm)-23(1.56 \mathrm{~g}, 4.66 \mathrm{mmol})$ in anhydrous THF $(20 \mathrm{~mL}$, $0.23 \mathrm{M}$ ) was added $n$-butyllithium ( $n$-BuLi, $3.49 \mathrm{~mL}, 1.6 \mathrm{M}$ solution in hexanes, $5.59 \mathrm{mmol})$ dropwise under argon atmosphere. The reaction mixture was stirred at the same temperature for 30 minutes, and gradually warmed to $0{ }^{\circ} \mathrm{C}$. Methyl chloroformate $(510 \mu \mathrm{L}, 6.52 \mathrm{mmol})$ was added dropwise and the resulting solution was stirred at $0{ }^{\circ} \mathrm{C}$ for 3 hours, then quenched with saturated aqueous $\mathrm{NH}_{4} \mathrm{Cl}$ solution $(20 \mathrm{~mL})$ and diluted with EtOAc $(30 \mathrm{~mL})$. The layers were separated and the aqueous layer was extracted with EtOAc $(2 \times 20 \mathrm{~mL})$. The combined organic layers were washed successively with $\mathrm{H}_{2} \mathrm{O}(5 \mathrm{~mL})$ and brine $(5 \mathrm{~mL})$, dried over anhydrous $\mathrm{MgSO}_{4}$ and concentrated in vacuo. The residue was purified by column chromatography (silica gel, heptane:EtOAc $=50: 1)$ to afford $( \pm)-24(1.63 \mathrm{~g}, 89 \%)$ as a colorless oil.

${ }^{1} \mathrm{H}$ NMR $\left(300 \mathrm{MHz}, \mathrm{CDCl}_{3}\right) \delta 7.24(\mathrm{~d}, J=1.2 \mathrm{~Hz}, 1 \mathrm{H}), 6.14(\mathrm{~d}, J=1.2 \mathrm{~Hz}, 1 \mathrm{H}), 4.79(\mathrm{t}, J=$ $7.2 \mathrm{~Hz}, 1 \mathrm{H}), 3.75(\mathrm{~s}, 3 \mathrm{H}), 2.35-2.25(\mathrm{~m}, 2 \mathrm{H}), 2.02(\mathrm{~s}, 3 \mathrm{H}), 2.00-1.88(\mathrm{~m}, 2 \mathrm{H}), 1.61-1.51(\mathrm{~m}$, $1 \mathrm{H}), 1.50-1.40(\mathrm{~m}, 1 \mathrm{H}), 1.04-0.92(\mathrm{~m}, 21 \mathrm{H}) ;{ }^{13} \mathrm{C} \mathrm{NMR}\left(75 \mathrm{MHz}, \mathrm{CDCl}_{3}\right) \delta 154.2,151.0,140.5$, 115.4, 112.9, 89.5, 73.0, 66.6, 52.5, 36.7, 23.5, 18.6, 18.0, 17.8, 12.3, 10.0; HRMS (ESI-TOF) $m / z:[\mathrm{M}+\mathrm{Na}]^{+}$Calcd for $\mathrm{C}_{22} \mathrm{H}_{36} \mathrm{NaO}_{4} \mathrm{Si} 415.2281$; Found 415.2288.

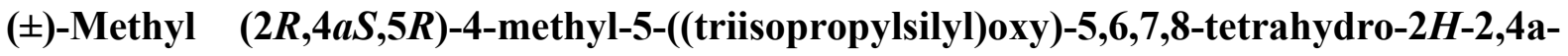
epoxynaphthalene-1-carboxylate $[( \pm)-25]$ 


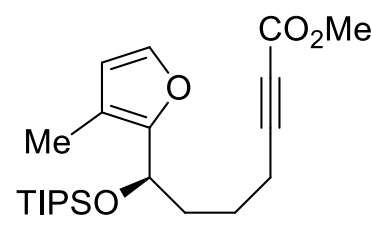

$( \pm)-24$

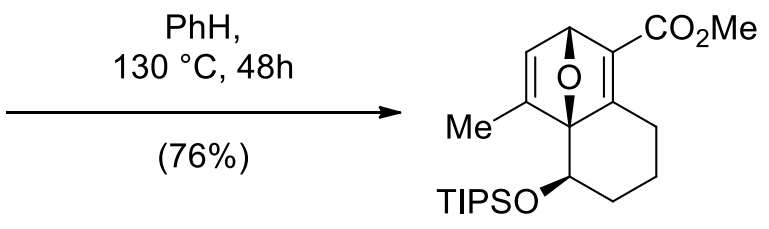

$( \pm)-25$

(Single Diastereomer)

A stirred solution of $( \pm)-24(350 \mathrm{mg}, 0.89 \mathrm{mmol})$ in benzene $(50 \mathrm{~mL}, 0.02 \mathrm{M})$ was heated to $130{ }^{\circ} \mathrm{C}$ for 48 hours under argon atmosphere. The reaction mixture was concentrated in vacuo. The residue was purified by column chromatography ( silica gel, heptane:EtOAc $=15: 1$ ) to afford ( \pm )-25 (266 mg, 76\%, single diastereomer) as a colorless oil.

${ }^{1} \mathrm{H}$ NMR $\left(300 \mathrm{MHz}, \mathrm{CDCl}_{3}\right) \delta 6.58(\mathrm{~d}, J=0.9 \mathrm{~Hz}, 1 \mathrm{H}), 5.44(\mathrm{~d}, J=1.5 \mathrm{~Hz}, 1 \mathrm{H}), 4.26(\mathrm{dd}, J=$ $12.0,4.8 \mathrm{~Hz}, 1 \mathrm{H}), 3.73(\mathrm{~s}, 3 \mathrm{H}), 3.38(\mathrm{~d}, J=19.2 \mathrm{~Hz}, 1 \mathrm{H}), 2.10(\mathrm{~d}, J=1.8 \mathrm{~Hz}, 3 \mathrm{H}), 2.20-1.89$ $(\mathrm{m}, 3 \mathrm{H}), 1.86-1.69(\mathrm{~m}, 1 \mathrm{H}), 1.59-1.42(\mathrm{~m}, 1 \mathrm{H}), 1.19-0.95(\mathrm{~m}, 21 \mathrm{H}) ;{ }^{13} \mathrm{C}$ NMR $(75 \mathrm{MHz}$, $\left.\mathrm{CDCl}_{3}\right) \delta 170.7,164.9,153.6,140.6,138.2,98.5,81.1,73.0,51.2,32.8,26.7,22.3,18.04$, 17.98, 14.6, 12.4; HRMS (ESI-TOF) $\mathrm{m} / z$ : $[\mathrm{M}+\mathrm{H}]^{+}$Calcd for $\mathrm{C}_{22} \mathrm{H}_{37} \mathrm{O}_{4} \mathrm{Si}$ 393.2461; Found 393.2458 .

\section{$( \pm)-(R)$-Furan-2-ylmethyl 7-(3-Methylfuran-2-yl)-7-((triisopropylsilyl)oxy)hept-2-ynoate} $[( \pm)-11]$

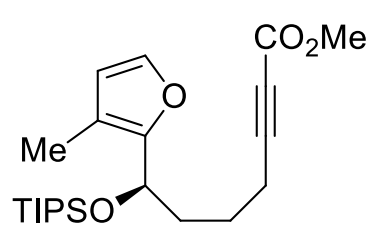

$( \pm)-24$
1) $\mathrm{LiOH}, \mathrm{THF} / \mathrm{H}_{2} \mathrm{O}(1: 1), \mathrm{rt}, 24 \mathrm{~h}$

2) 18, HATU, DIPEA, $\mathrm{DCM}, 0{ }^{\circ} \mathrm{C}$ to $\mathrm{rt}, 3 \mathrm{~h}$

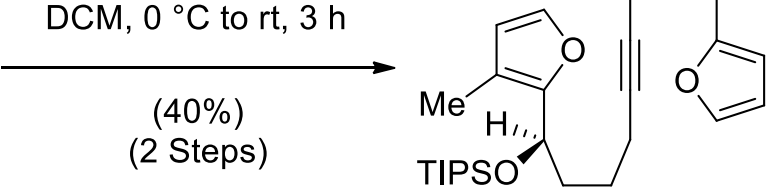

$( \pm)-11$

To a stirred solution of $( \pm)-\mathbf{2 4}(300 \mathrm{mg}, 0.760 \mathrm{mmol})$ in $\mathrm{THF} / \mathrm{H}_{2} \mathrm{O}(3.0 \mathrm{~mL}, \mathrm{v}: \mathrm{v}=1: 1,0.26 \mathrm{M})$ was added lithium hydroxide ( $\mathrm{LiOH}, 46.0 \mathrm{mg}, 1.91 \mathrm{mmol})$ at room temperature. The reaction mixture was stirred at the same temperature for 24 hours, then the $\mathrm{pH}$ of the solution was adjusted to 2 by the addition of $1 \mathrm{~N} \mathrm{HCl}$ solution dropwise and diluted with EtOAc $(10 \mathrm{~mL})$. The layers were separated and the aqueous layer was extracted with EtOAc $(2 \times 10 \mathrm{~mL})$. The combined organic layers were washed successively with $\mathrm{H}_{2} \mathrm{O}(5 \mathrm{~mL})$ and brine $(5 \mathrm{~mL})$, dried over anhydrous $\mathrm{MgSO}_{4}$ and concentrated in vacuo to afford the corresponding carboxylic acid as a colorless oil, which was used directly in the next step without further purification.

To a cooled $\left(0{ }^{\circ} \mathrm{C}\right)$ stirred solution of the carboxylic acid prepared above $(289 \mathrm{mg}, 0.763 \mathrm{mmol})$ in $\mathrm{MeCN}(3.5 \mathrm{~mL}, 0.22 \mathrm{M}$ ) was successively added 1-[bis(dimethylamino)methylene]-1H1,2,3-triazolo[4,5-b]pyridinium 3-oxide hexafluorophosphate (HATU, $435 \mathrm{mg}, 1.20 \mathrm{mmol}$ ), DIPEA $(170 \mu \mathrm{L}, 990 \mu \mathrm{mol})$ and furfuryl alcohol $(70.0 \mu \mathrm{L}, 840 \mu \mathrm{mol})$ under argon atmosphere. The reaction mixture was gradually warmed to room temperature over the period of 3 hours, then quenched with $\mathrm{H}_{2} \mathrm{O}(3 \mathrm{~mL})$ and diluted with EtOAc $(6 \mathrm{~mL})$. The layers were separated and the aqueous layer was extracted with EtOAc $(2 \times 10 \mathrm{~mL})$. The combined organic layers were washed successively with $\mathrm{H}_{2} \mathrm{O}(5 \mathrm{~mL})$ and brine $(5 \mathrm{~mL})$, dried over anhydrous $\mathrm{MgSO}_{4}$ 
and concentrated in vacuo. The residue was purified by column chromatography (silica gel, heptane:EtOAc $=20: 1)$ to afford $( \pm)-\mathbf{1 1}(140 \mathrm{mg}, 40 \%$ over 2 steps $)$ as a colorless oil.

${ }^{1} \mathrm{H}$ NMR $\left(600 \mathrm{MHz}, \mathrm{CDCl}_{3}\right) \delta 7.45(\mathrm{~d}, J=1.8 \mathrm{~Hz}, 1 \mathrm{H}), 7.25(\mathrm{~d}, J=1.8 \mathrm{~Hz}, 1 \mathrm{H}), 6.46(\mathrm{~d}, J=$ $3.2 \mathrm{~Hz}, 1 \mathrm{H}), 6.41-6.35(\mathrm{~m}, 1 \mathrm{H}), 6.15(\mathrm{~d}, J=1.6 \mathrm{~Hz}, 1 \mathrm{H}), 5.14(\mathrm{~s}, 2 \mathrm{H}), 4.79(\mathrm{t}, J=6.8 \mathrm{~Hz}, 1 \mathrm{H})$, 2.37-2.34 (m, 2H), $2.02(\mathrm{~s}, 3 \mathrm{H}), 2.01-1.87(\mathrm{~m}, 2 \mathrm{H}), 1.68-1.51(\mathrm{~m}, 1 \mathrm{H}), 1.51-1.36(\mathrm{~m}, 1 \mathrm{H})$, $1.06-0.91(\mathrm{~m}, 21 \mathrm{H}) ;{ }^{13} \mathrm{C}$ NMR $\left(75 \mathrm{MHz}, \mathrm{CDCl}_{3}\right) \delta 153.5,151.1,148.7,143.6,140.6,115.5$, 113.0, 111.5, 110.8, 90.2, 73.0, 66.7, 59.1, 36.9, 23.6, 18.8, 18.1, 17.9, 12.4, 10.1; HRMS (ESIquadrupole) $m / z$ : $[\mathrm{M}+\mathrm{H}]^{+}$Calcd for $\mathrm{C}_{26} \mathrm{H}_{39} \mathrm{O}_{5} \mathrm{Si}$ 459.2566; Found 459.2565.

\section{( \pm )-(R)- $N$-Benzyl- $N$-(furan-2-ylmethyl)-7-(3-methylfuran-2-yl)-7- ((triisopropylsilyl)oxy)hept-2-ynamide $[( \pm)-27]$}

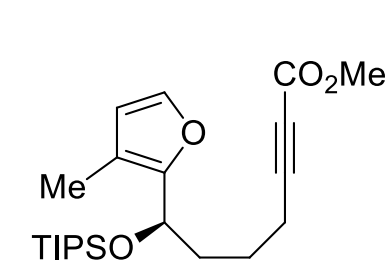

$( \pm)-24$
1) $\mathrm{LiOH}, \mathrm{THF} / \mathrm{H}_{2} \mathrm{O}(1: 1), \mathrm{rt}, 24 \mathrm{~h}$

2) 26, HATU, DIPEA, $\mathrm{MeCN}, 0^{\circ} \mathrm{C}$ to $\mathrm{rt}, 3 \mathrm{~h}$

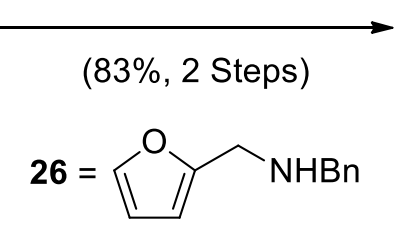

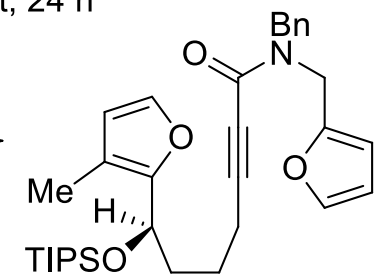

$( \pm)-27$

To a stirred solution of $( \pm)-24(519 \mathrm{mg}, 1.32 \mathrm{mmol})$ in $\mathrm{THF} / \mathrm{H}_{2} \mathrm{O}(5.2 \mathrm{~mL}$, v:v=1:1, $0.26 \mathrm{M})$ was added $\mathrm{LiOH}(80.0 \mathrm{mg}, 1.91 \mathrm{mmol})$ at room temperature. The reaction mixture was stirred at the same temperature for 24 hours, then the $\mathrm{pH}$ of the solution was adjusted to 2 by the addition of $1 \mathrm{~N} \mathrm{HCl}$ solution dropwise and diluted with EtOAc $(15 \mathrm{~mL})$. The layers were separated and the aqueous layer was extracted with EtOAc $(2 \times 15 \mathrm{~mL})$. The combined organic layers were washed successively with $\mathrm{H}_{2} \mathrm{O}(5 \mathrm{~mL})$ and brine $(5 \mathrm{~mL})$, dried over anhydrous $\mathrm{MgSO}_{4}$ and concentrated in vacuo to afford the corresponding carboxylic acid as a colorless oil, which was used directly in the next step without further purification.

To a cooled $\left(0{ }^{\circ} \mathrm{C}\right)$ stirred solution of the carboxylic acid prepared above $(500 \mathrm{mg}, 1.32 \mathrm{mmol})$ in $\mathrm{MeCN}(6.0 \mathrm{~mL}, 0.22 \mathrm{M})$ was successively added 1-[bis(dimethylamino)methylene]-1H1,2,3-triazolo[4,5-b]pyridinium 3-oxide hexafluorophosphate (HATU, $750 \mathrm{mg}, 1.98 \mathrm{mmol}$ ), $N, N$-diisopropylamine (DIPEA, $300 \mu \mathrm{L}, 1.72 \mathrm{mmol}$ ) and $N$-benzyl-1-(furan-2-yl)methanamine $(26)^{2}(270 \mathrm{mg}, 1.45 \mathrm{mmol})$ under argon atmosphere. The reaction mixture was gradually warmed to room temperature over the period of 3 hours, then quenched with $\mathrm{H}_{2} \mathrm{O}(10 \mathrm{~mL})$ and diluted with EtOAc $(20 \mathrm{~mL})$. The layers were separated and the aqueous layer was extracted with EtOAc $(2 \times 20 \mathrm{~mL})$. The combined organic layers were washed successively with $\mathrm{H}_{2} \mathrm{O}$ $(80 \mathrm{~mL})$ and brine, dried over anhydrous $\mathrm{MgSO}_{4}$ and concentrated in vacuo. The residue was purified by column chromatography (silica gel, heptane:EtOAc $=20: 1)$ to afford $( \pm)-27(600$ $\mathrm{mg}, 83 \%$ over 2 steps) as a colorless oil.

${ }^{1} \mathrm{H}$ NMR $\left(600 \mathrm{MHz}, \mathrm{CDCl}_{3}\right) \delta 7.38-7.16(\mathrm{~m}, 7 \mathrm{H}), 6.31(\mathrm{dd}, J=6.0,1.8 \mathrm{~Hz}, 1 \mathrm{H}), 6.21(\mathrm{t}, J=$ $3.6 \mathrm{~Hz}, 1 \mathrm{H}), 6.11(\mathrm{~d}, J=8.8 \mathrm{~Hz}, 1 \mathrm{H}), 4.80-4.74(\mathrm{~m}, 1 \mathrm{H}), 4.73(\mathrm{~s}, 1 \mathrm{H}), 4.60-4.50(\mathrm{~m}, 2 \mathrm{H}), 4.46$ (s, 1H), 2.37 (dt, $J=7.2,3.2 \mathrm{~Hz}, 1 \mathrm{H}), 2.31(\mathrm{dt}, J=7.2,3.2 \mathrm{~Hz}, 1 \mathrm{H}), 1.98-1.97(\mathrm{~m}, 3 \mathrm{H}), 1.96-$ $1.82(\mathrm{~m}, 2 \mathrm{H}), 1.67-1.56(\mathrm{~m}, 1 \mathrm{H}), 1.50-1.34(\mathrm{~m}, 1 \mathrm{H}), 1.04-0.85(\mathrm{~m}, 21 \mathrm{H}) ;{ }^{13} \mathrm{C} \mathrm{NMR}(150 \mathrm{MHz}$, 
$\left.\mathrm{CDCl}_{3}\right) \delta 154.8,151.1,150.7,142.7,140.4,136.4,128.8,127.7,115.3,112.9,110.4,109.1$, 93.6, 74.1, 66.6, 51.8, 46.6, 44.4, 39.2, 36.9, 23.9, 19.0, 18.0, 12.3, 9.9; HRMS (ESI-TOF) $\mathrm{m} / \mathrm{z}$ : $[\mathrm{M}+\mathrm{Na}]^{+}$Calcd for $\mathrm{C}_{33} \mathrm{H}_{45} \mathrm{NNaO}_{4} \mathrm{Si} 570.3016$; Found 570.3003 .

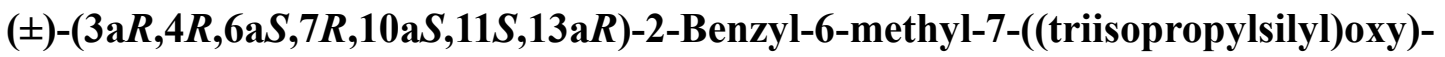
4,7,8,9,10,11-hexahydro-1H-4,6a:11,13a-diepoxynaphtho[1,8a-d]isoindol-3(2H)-one $[( \pm)$ 28]

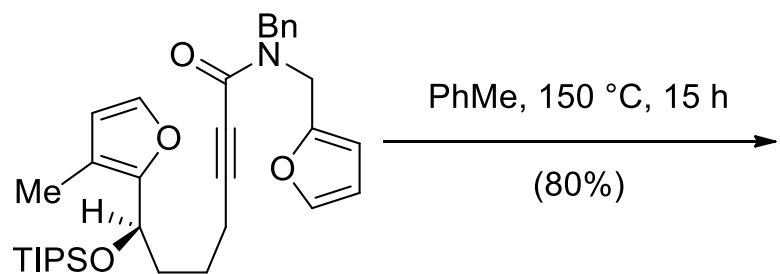

$( \pm)-27$

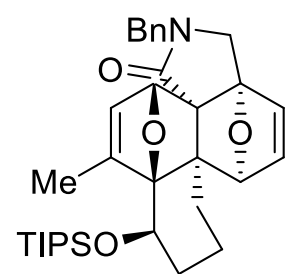

$( \pm)-28$

(Single Diastereomer)

A stirred solution of $( \pm)-27(560 \mathrm{mg}, 1.02 \mathrm{mmol})$ in toluene $(50 \mathrm{~mL}, 0.02 \mathrm{M})$ was heated to $150{ }^{\circ} \mathrm{C}$ for 15 hours under argon atmosphere. The reaction mixture was concentrated in vacuo. The residue was purified by column chromatography (silica gel, heptane:EtOAc $=20: 1$ ) to afford $( \pm)$-28 (450 mg, 80\%, single diastereomer) as a white solid.

m.p. $160-162{ }^{\circ} \mathrm{C} ;{ }^{1} \mathrm{H}$ NMR (600 MHz, $\left.\mathrm{CDCl}_{3}\right) \delta$ 7.35-7.24 (m, 3H), 7.22-7.16 (m, 2H), 6.51 $(\mathrm{dd}, J=6.0,1.2 \mathrm{~Hz}, 1 \mathrm{H}), 6.24(\mathrm{~d}, J=6.0 \mathrm{~Hz}, 1 \mathrm{H}), 6.19(\mathrm{~s}, 1 \mathrm{H}), 4.81(\mathrm{~d}, J=1.2 \mathrm{~Hz}, 1 \mathrm{H}), 4.61$ $(\mathrm{d}, J=15.0 \mathrm{~Hz}, 1 \mathrm{H}), 4.39(\mathrm{~d}, J=15.0 \mathrm{~Hz}, 1 \mathrm{H}), 4.11(\mathrm{~d}, J=1.2 \mathrm{~Hz}, 1 \mathrm{H}), 4.02(\mathrm{dd}, J=10.2,7.2$ $\mathrm{Hz}, 1 \mathrm{H}), 3.70$ (d, $J=12.0 \mathrm{~Hz}, 1 \mathrm{H}), 3.43(\mathrm{~d}, J=12.0 \mathrm{~Hz}, 1 \mathrm{H}), 2.19$ (s, 3H), 2.10-1.79 (m, 6H), $1.05(\mathrm{~m}, 21 \mathrm{H}) ;{ }^{13} \mathrm{C} \mathrm{NMR}\left(150 \mathrm{MHz}, \mathrm{CDCl}_{3}\right) \delta 171.9,150.9,139.6,136.2,134.2,129.8,128.8$, 127.7, 127.5, 92.6, 91.0, 83.4, 77.5, 70.2, 66.8, 66.4, 47.8, 46.6, 31.7, 29.0, 21.6, 18.9, 18.1, 18.0, 12.3; HRMS (ESI-TOF) $\mathrm{m} / z$ : $[\mathrm{M}+\mathrm{Na}]^{+}$Calcd for $\mathrm{C}_{33} \mathrm{H}_{45} \mathrm{NNaO}_{4} \mathrm{Si}$ 570.3016; Found 570.3019 .

$( \pm)-(3 a R, 4 R, 6 R, 6 a S, 7 R, 10 a S, 11 S, 13 a R)-2-B e n z y l-7-h y d r o x y-6-m e t h y l d e c a h y d r o-1 H-$ 4,6a:11,13a-diepoxynaphtho[1,8a-d]isoindol-3(2H)-one $[( \pm)-29]$

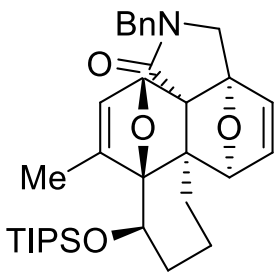

$( \pm)-28$

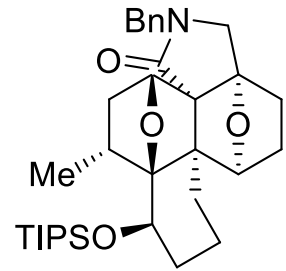

$( \pm)-29$

To a stirred solution of $( \pm)-\mathbf{2 8}(110 \mathrm{mg}, 0.20 \mathrm{mmol})$ in anhydrous EtOAc/MeOH $(3.5 \mathrm{~mL}$, $\mathrm{v}: \mathrm{v}=2.5: 1,0.06 \mathrm{M})$ was added $\mathrm{Pd} / \mathrm{C}(10 \% \mathrm{wt}, 44.0 \mathrm{mg}, 40.0 \mu \mathrm{mol})$. The reaction mixture was stirred at room temperature for 72 hours under hydrogen atmosphere, then filtered through a pad of Celite 545 and the filtrate was concentrated in vacuo. The residue was purified by 
column chromatography ( single diastereomer) as a white solid.

m.p. $160-162{ }^{\circ} \mathrm{C} ;{ }^{1} \mathrm{H}$ NMR $\left(600 \mathrm{MHz}, \mathrm{CDCl}_{3}\right) \delta 7.33(\mathrm{t}, J=7.4 \mathrm{~Hz}, 2 \mathrm{H}), 7.28-7.23(\mathrm{~m}, 1 \mathrm{H})$, $7.20(\mathrm{~d}, J=7.4 \mathrm{~Hz}, 2 \mathrm{H}), 4.6(\mathrm{~d}, J=15.0 \mathrm{~Hz}, 1 \mathrm{H}), 4.42-4.35(\mathrm{~m}, 2 \mathrm{H}), 4.20-4.13(\mathrm{~m}, 2 \mathrm{H}), 3.39$ (q, $J=10.6 \mathrm{~Hz}, 2 \mathrm{H}), 2.88-2.78(\mathrm{~m}, 1 \mathrm{H}), 2.46(\mathrm{dt}, J=6.4 \mathrm{~Hz}, 1 \mathrm{H}, 2.37-2.30(\mathrm{~m}, 1 \mathrm{H}), 2.00$ $1.40(\mathrm{~m}, 15 \mathrm{H}), 1.08(\mathrm{~m}, 21 \mathrm{H}) ;{ }^{13} \mathrm{C} \mathrm{NMR}\left(150 \mathrm{MHz}, \mathrm{CDCl}_{3}\right) \delta 172.5,136.2,128.8,127.7$, 127.5, 91.2, 90.7, 84.8, 70.7, 66.6, 66.5, 48.4, 46.4, 34.9, 33.2, 32.3, 27.8, 26.4, 26.2, 21.4, 19.0, 18.1, 18.0, 12.5; HRMS (ESI-TOF) $m / z$ : $[\mathrm{M}+\mathrm{Na}]^{+} \mathrm{Calcd}$ for $\mathrm{C}_{33} \mathrm{H}_{49} \mathrm{NNaO}_{4} \mathrm{Si}$ 574.3329; Found 574.3307 .

\section{( \pm )-(3a $R, 4 R, 6 R, 6 \mathrm{a} S, 7 R, 10 \mathrm{a} S, 11 S, 13 \mathrm{a} R)-2-B e n z y l-7-h y d r o x y-6-m e t h y l d e c a h y d r o-1 H$ -} 4,6a:11,13a-diepoxynaphtho[1,8a-d]isoindol-3(2H)-one $[( \pm)-30]$

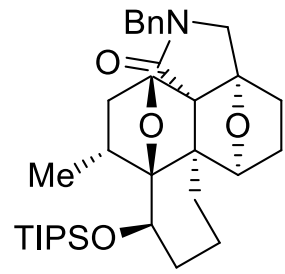

$( \pm)-29$

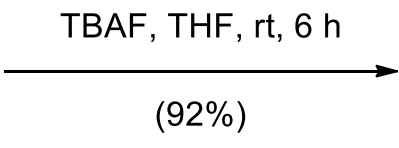

$(92 \%)$

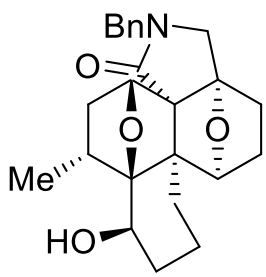

$( \pm)-30$

To a stirred solution of $( \pm)-29(61.0 \mathrm{mg}, 0.110 \mathrm{mmol})$ in THF $(1.0 \mathrm{~mL}, 0.11 \mathrm{M})$ was added tetrabutylammonium fluoride (TBAF, $170 \mu \mathrm{L}, 1 \mathrm{M}$ solution in THF, $170 \mu \mathrm{mol}$ ) dropwise at room temperature under argon atmosphere. The reaction mixture was stirred at the same temperature for 6 hours, then quenched with saturated aqueous $\mathrm{NH}_{4} \mathrm{Cl}$ solution $(1 \mathrm{~mL})$ and diluted with EtOAc $(5 \mathrm{~mL})$. The layers were separated and the aqueous layer was extracted with EtOAc $(2 \times 5 \mathrm{~mL})$. The combined organic layers were washed successively with $\mathrm{H}_{2} \mathrm{O}(2$ $\mathrm{mL})$ and brine $(2 \mathrm{~mL})$, dried over anhydrous $\mathrm{MgSO}_{4}$ and concentrated in vacuo. The residue was purified by column chromatography (silica gel, heptane:EtOAc $=5: 1$ ) to afford $( \pm)$-30 (40.0 $\mathrm{mg}, 92 \%)$ as a white crystal.

m.p. $160-162{ }^{\circ} \mathrm{C} ;{ }^{1} \mathrm{H}$ NMR $\left(300 \mathrm{MHz}, \mathrm{CDCl}_{3}\right) \delta 7.36-7.30(\mathrm{~m}, 3 \mathrm{H}), 7.23-7.21(\mathrm{~m}, 2 \mathrm{H}), 4.60$ $(\mathrm{d}, J=15.0 \mathrm{~Hz}, 1 \mathrm{H}), 4.46-4.41(\mathrm{~m}, 2 \mathrm{H}), 4.29-4.27(\mathrm{~m}, 1 \mathrm{H}), 4.15-4.12(\mathrm{~m}, 1 \mathrm{H}), 3.48-3.39$ (m, 2H), $2.72(\mathrm{~d}, J=9.6 \mathrm{~Hz}, 1 \mathrm{H}), 2.54-2.49(\mathrm{~m}, 1 \mathrm{H}), 2.35-2.28(\mathrm{~m}, 1 \mathrm{H}), 2.07-1.96(\mathrm{~m}, 4 \mathrm{H}), 1.96-$ $1.85(\mathrm{~m}, 4 \mathrm{H}), 1.64-1.61(\mathrm{~m}, 4 \mathrm{H}), 1.56-1.48(\mathrm{~m}, 2 \mathrm{H}), 1.25(\mathrm{~s}, 1 \mathrm{H}) ;{ }^{13} \mathrm{C} \mathrm{NMR}\left(75 \mathrm{MHz}, \mathrm{CDCl}_{3}\right)$ $\delta 172.2,136.0,128.8,127.7,127.6,90.9,90.7,84.9,78.3,70.1,66.5,66.4,48.4,46.4,34.9$, $33.2,30.2,27.9,26.3,26.3,21.3,19.2$; HRMS (ESI-TOF) $m / z$ : $[\mathrm{M}+\mathrm{Na}]^{+}$Calcd for $\mathrm{C}_{24} \mathrm{H}_{30} \mathrm{NO}_{4}$ 396.2175; Found 396.2141. 


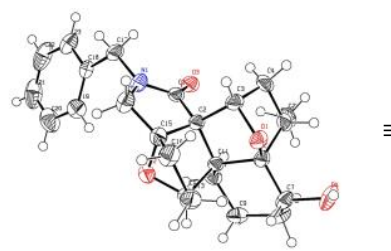

ORTEP Diagram of $( \pm)-30$

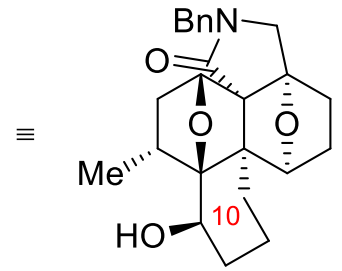

$( \pm)-30$
Empirical formula

Formula weight

Temperature

Wavelength

Crystal system

Space group

Unit cell dimensions

Volume

Z

Density (calculated)

Absorption coefficient

$\mathrm{F}(000)$

Crystal size

Theta range for data collection

Index ranges

Reflections collected

Independent reflections

Completeness to theta $=25.242^{\circ}$

Absorption correction

Max. and min. transmission

Refinement method

Data / restraints / parameters

Goodness-of-fit on $\mathrm{F}^{2}$

Final $\mathrm{R}$ indices [I>2sigma(I)]

$\mathrm{R}$ indices (all data)

Extinction coefficient

Largest diff. peak and hole
$\mathrm{C}_{24} \mathrm{H}_{29} \mathrm{NO}_{4}$

395.48

223(2) K

$0.71073 \AA$

Monoclinic

$\mathrm{P} 21 / \mathrm{c}$

$\mathrm{a}=7.3323(5) \AA \quad \alpha=90^{\circ}$.

$\mathrm{b}=10.8726(7) \AA \quad \beta=91.762(2)^{\circ}$.

$\mathrm{c}=25.0071(15) \AA \gamma=90^{\circ}$.

1992.7(2) $\AA^{3}$

4

$1.318 \mathrm{Mg} / \mathrm{m}^{3}$

$0.089 \mathrm{~mm}^{-1}$

848

$0.170 \times 0.120 \times 0.100 \mathrm{~mm}^{3}$

2.043 to $28.312^{\circ}$.

$-9<=\mathrm{h}<=9,-14<=\mathrm{k}<=14,-33<=\mathrm{l}<=33$

65789

$4979[\mathrm{R}($ int $)=0.1068]$

$100.0 \%$

Semi-empirical from equivalents

0.7457 and 0.7025

Full-matrix least-squares on $\mathrm{F}^{2}$ 4979 / 0 / 264

1.011

$\mathrm{R} 1=0.0495, \mathrm{wR} 2=0.1034$

$\mathrm{R} 1=0.0923, \mathrm{wR} 2=0.1207$

$\mathrm{n} / \mathrm{a}$

0.276 and -0.184 e. $\AA^{-3}$ 


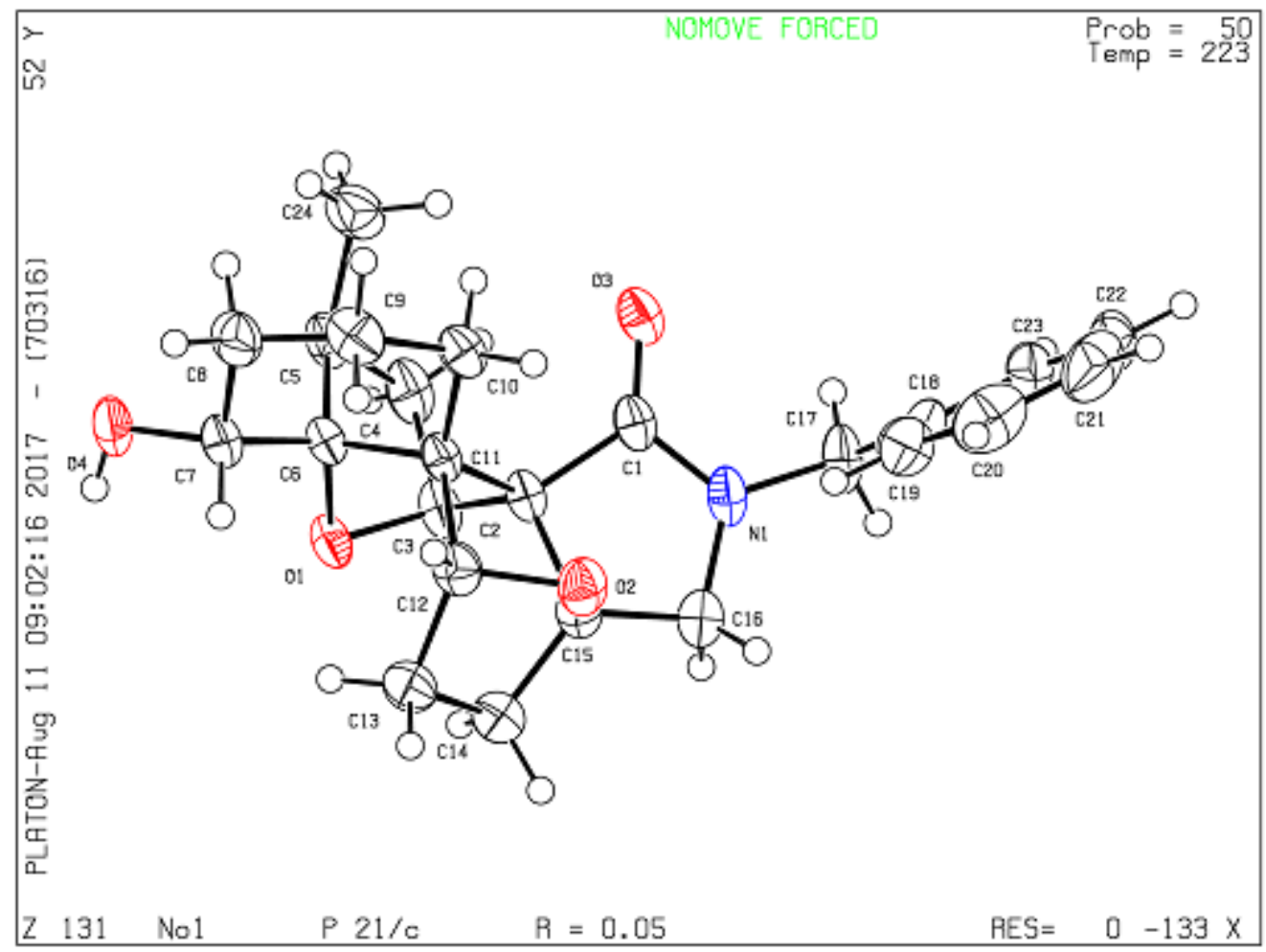




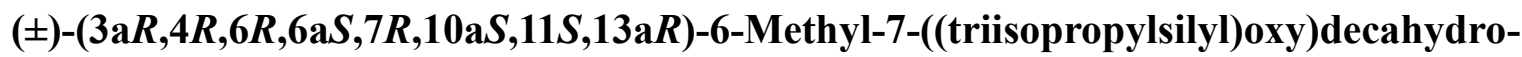
$1 H-4,6 a: 11,13 a-d i e p o x y n a p h t h o[1,8 a-d]$ isoindol-3(2H)-one $[( \pm)-31]$

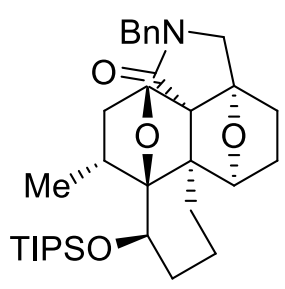

$( \pm)-29$

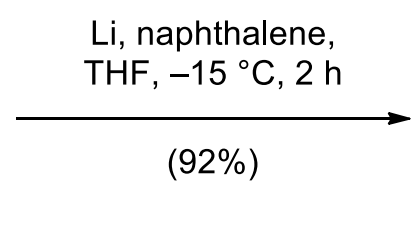

$\mathrm{Li}$, naphthalene,

$(92 \%)$

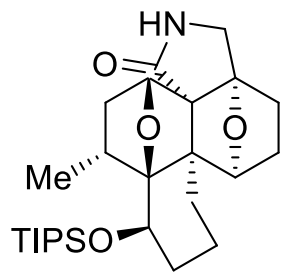

$( \pm)-31$

A stirred solution of naphthalene $(140 \mathrm{mg}, 1.09 \mathrm{mmol})$ in anhydrous THF $(5 \mathrm{~mL}, 0.05 \mathrm{M})$ was added lithium (Li) metal $(6.00 \mathrm{mg}, 0.820 \mathrm{mmol})$ portionwise in small pieces. The solution was stirred at room temperature under argon atmosphere until lithium (Li) metal was completely dissolved (about 3 hours). The resulting dark green solution of lithium naphthalenide ${ }^{3}$ was then cooled to $-15{ }^{\circ} \mathrm{C}$, followed by the addition of a solution of $( \pm)-29(0.150 \mathrm{mg}, 0.270 \mathrm{mmol})$ in anhydrous THF $(2.0 \mathrm{~mL})$ dropwise over 5 minutes. The reaction mixture was stirred at the same temperature for 2 hours, then quenched with saturated aqueous $\mathrm{NH}_{4} \mathrm{Cl}$ solution $(5 \mathrm{~mL})$ and diluted with EtOAc $(10 \mathrm{~mL})$. The layers were separated and the aqueous layer was extracted with EtOAc $(2 \times 10 \mathrm{~mL})$. The combined organic layers were washed successively with $\mathrm{H}_{2} \mathrm{O}(5 \mathrm{~mL})$ and brine $(5 \mathrm{~mL})$, dried over anhydrous $\mathrm{MgSO}_{4}$ and concentrated in vacuo. The residue was purified by column chromatography (silica gel, heptane:EtOAc $=5: 1$ ) to afford $( \pm)$-31 (115 mg, 92\%) as a white solid.

m.p. $160-162{ }^{\circ} \mathrm{C} ;{ }^{1} \mathrm{H}$ NMR $\left(600 \mathrm{MHz}, \mathrm{CDCl}_{3}\right) \delta 5.61(\mathrm{~s}, 1 \mathrm{H}), 4.40(\mathrm{~d}, J=5.4 \mathrm{~Hz}, 1 \mathrm{H}), 4.2(\mathrm{~d}$, $J=5.4 \mathrm{~Hz}, 1 \mathrm{H}), 4.18-4.13(\mathrm{~m}, 1 \mathrm{H}), 3.57(\mathrm{~d}, J=11.6 \mathrm{~Hz}, 1 \mathrm{H}), 3.47(\mathrm{~d}, J=11.6 \mathrm{~Hz}, 1 \mathrm{H}), 2.86-$ $2.76(\mathrm{~m}, 1 \mathrm{H}), 2.45-2.29(\mathrm{~m}, 2 \mathrm{H}), 1.98-1.6(\mathrm{~m}, 9 \mathrm{H}), 1.51(\mathrm{~d}, J=7.5 \mathrm{~Hz}, 3 \mathrm{H}), 1.48-1.43(\mathrm{~m}$, $1 \mathrm{H}), 1.08(\mathrm{~m}, 21 \mathrm{H}) ;{ }^{13} \mathrm{C} \mathrm{NMR}\left(150 \mathrm{MHz}, \mathrm{CDCl}_{3}\right) \delta 175.6,93.6,91.2,84.9,70.6,66.6,66.0$, $44.1,34.9,33.0,32.2,27.7,26.7,26.1,21.4,19.1,18.1,18.0,12.5 ;{ }^{13} \mathrm{C} \mathrm{NMR}\left(150 \mathrm{MHz}, \mathrm{CDCl}_{3}\right)$ $\delta 175.6,93.6,91.2,84.9,70.6,66.6,66.0,44.1,34.9,33.0,32.2,27.7,26.7,26.1,21.4,19.1$, 18.1, 18.0, 12.5; HRMS (ESI-TOF) $m / z$ : $[\mathrm{M}+\mathrm{H}]^{+}$Calcd for $\mathrm{C}_{26} \mathrm{H}_{44} \mathrm{NO}_{4} \mathrm{Si}$ 462.3040; Found 462.3047 .

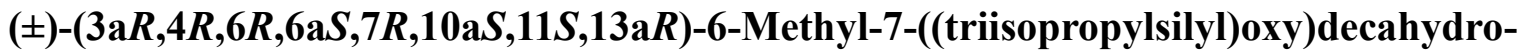

\section{H,4H,11H-4,6a:11,13a-diepoxy[1,8a-d]isobenzofuran-3-one $[( \pm)-15]$}

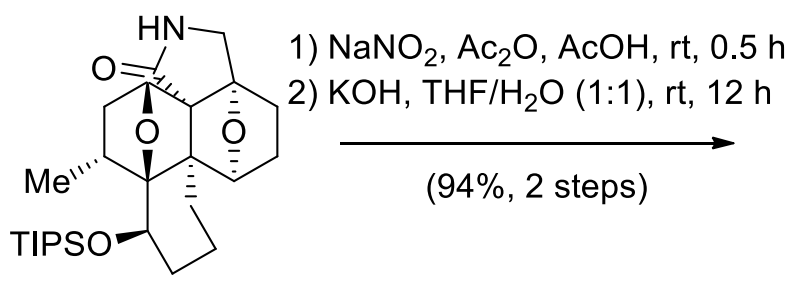

$( \pm)-31$

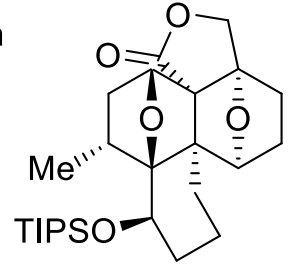

$( \pm)-15$

To a stirred solution of $( \pm)-31(70.0 \mathrm{mg}, 0.150 \mathrm{mmol})$ in acetic acid (AcOH, $0.35 \mathrm{~mL})$ and acetic anhydride $\left(\mathrm{Ac}_{2} \mathrm{O}, 1.16 \mathrm{~mL}\right)$ was added $\mathrm{NaNO}_{2}{ }^{4}(30.0 \mathrm{mg}, 0.460 \mathrm{mmol})$. The reaction 
mixture turned yellow immediately, and a brown gas escaped. The reaction mixture was stirred at room temperature for 30 minutes, then evaporated and the remaining solid was taken in EtOAc $(50 \mathrm{~mL})$ which was washed with saturated aqueous $\mathrm{NaHCO}_{3}$ solution $(4 \times 20 \mathrm{~mL})$. The combined organic layers were dried over anhydrous $\mathrm{Na}_{2} \mathrm{SO}_{4}$ and concentrated in vacuo. To remove residual acetic acid $(\mathrm{AcOH})$ from the crude product, the reaction mixture was concentrated after the addition of toluene, providing sufficiently pure $N$-nitrosolactam which was used in the next step without further purification.

To a stirred solution of this $N$-nitrosolactam prepared above in $\mathrm{THF} / \mathrm{H}_{2} \mathrm{O}(2.0 \mathrm{~mL}$, v:v=1:1, $0.08 \mathrm{M})$ was added $\mathrm{KOH}(17.2 \mathrm{mg}, 0.310 \mathrm{mmol})$ at room temperature. The reaction mixture was stirred at the same temperature for 12 hours, then quenched with $\mathrm{H}_{2} \mathrm{O}(10 \mathrm{~mL})$. The $\mathrm{pH}$ of the solution was adjusted to 2 by the addition of $1 \mathrm{~N} \mathrm{HCl}$ solution dropwise. The resulting precipitates were collected by a filteration through a filter paper. The resulting filter cake was rinsed with $\mathrm{H}_{2} \mathrm{O}$ and then it afforded $( \pm)-\mathbf{1 5}(67.0 \mathrm{mg}, 94 \%$ over 2 steps $)$ as a white solid.

m.p. $160-162{ }^{\circ} \mathrm{C} ;{ }^{1} \mathrm{H}$ NMR $\left(300 \mathrm{MHz}, \mathrm{CDCl}_{3}\right) \delta 4.52(\mathrm{~d}, J=11.1 \mathrm{~Hz}, 1 \mathrm{H}), 4.45(\mathrm{~d}, J=5.4 \mathrm{~Hz}$, $1 \mathrm{H}), 4.29(\mathrm{~d}, J=2.7 \mathrm{~Hz}, 1 \mathrm{H}), 4.26(\mathrm{~d}, J=2.7 \mathrm{~Hz}, 1 \mathrm{H}), 4.20-4.09(\mathrm{~m}, 1 \mathrm{H}), 2.92-2.75(\mathrm{~m}, 1 \mathrm{H})$, 2.41-2.24 (m, 2H), 2.07-1.54 (m, 9H), 1.53-1.42 (m, 4H), $1.08(\mathrm{~s}, 21 \mathrm{H}) ;{ }^{13} \mathrm{C}$ NMR $(75 \mathrm{MHz}$, $\left.\mathrm{CDCl}_{3}\right) \delta 174.9,93.6,91.4,85.4,70.4,68.4,68.2,64.8,34.9,32.8,32.1,27.2,26.1,25.3,21.2$, 19.3, 18.1, 18.0, 12.5; HRMS (EI-quadrupole) $\mathrm{m} / z$ : $[\mathrm{M}]^{+}$Calcd for $\mathrm{C}_{26} \mathrm{H}_{42} \mathrm{O}_{5} \mathrm{Si}$ : 462.2802; Found 462.2798. 


\section{References}

1. Tata, J. R.; Kevin, N. J.; Cheng, Y.; Zhang, F.; Kirk, A. B.; Raghavan, S.; Petrillo, D. E.; Varsolona, R. J.; Purick, R.; Hoerrner, R. S.; Askin, D.; Armstrong, J. D, III; Fleitz, F. J.; Lu, Z.; Huening, T.; Rano, T. A.; Chapman, K. T.; Duffy, J. L. U.S. Patent 6,642,237, 2003, B1.

2. Brice, H.; Clayden, J. Chem. Commun. 2009, 1964-1965.

3. (a) Liu, H. -J.; Yip, J.; Shia, K.-S. Tetrahedron Lett. 1997, 38, 2253-2256. (b) Huang, P. Q.; Zheng, X.; Wang, S. L.; Ye, J. L.; Jin, L. R.; Chen, Z. Tetrahedron: Asymmetry 1999, 10, 33093317.

4. (a) Benningshof, J. C.; Blaauw, R. H.; van Ginkel, A. E.; van Maarseveen, J. H.; Rutjes, F. P. J. T.; Hiemstra, H. J. Chem. Soc., Perkin Trans. I 2002, 1693-1700. (b) Wang, C.-C.; Li, W.D. Z. J. Org. Chem. 2012, 77, 4217-4225. 